W. Robert J. Funnell \& Charles A. Laszlo

\title{
Modeling of the cat eardrum as a thin shell using thefinite-element method
}

Published in:

Journal of the Acoustical Society of America, 63(5), May 1978, pp.

1461-1467

Copyright (1978) Acoustical Society of America. This article may be downloaded for personal use only. Any other use requires prior permission of the author and the Acoustical Society of America.

doi:10.1121/1.381892

http://scitation.aip.org/content/asa/journal/jasa/63/5/10.1121/1.381892 


\title{
Modeling of the cat eardrum as a thin shell using the finite-element method
}

\author{
W. Robert J. Funnell and Charles A. Laszlo \\ BioMedical Engineering Unit \& Department of Otolaryngology, McGill University, Montréal, \\ Québec, Canada \\ (Received 20 April 1977; revised 31 August 1977)
}

\begin{abstract}
A finite-element model of the cat eardrum is presented which includes the effects of the three-dimensional curved conical shape of the drum. The model is valid at low frequencies (below $1-2 \mathrm{kHz}$ ) and within the range of linear vibration amplitudes. The material properties used are based on a review of the literature. The critical material parameters are the stiffness $\left(2 \times 10^{8} \mathrm{dyn}^{-2}\right)$ and thickness $(40 \mu \mathrm{m})$ of the pars tensa. The model exhibits a vibration pattern and amplitude very similar to those observed experimentally using laser holography. A number of parameters are varied in order to study their relative importance in the model.
\end{abstract}

PACS numbers: 43.63.Bq, 43.63.Hx, 43.40.Ey

\section{INTRODUCTION}

Presently available models of the eardrum are inadequate either to provide insight into the principles of the mechanical operation of the drum, or to permit quantitative analysis of pathological conditions as an aid in the planning and evaluation of corrective techniques. In a thesis (Funnell, 1975) and two conference papers (Funnell and Laszlo, 1974, 1975) we have introduced the use of the finite-element method as a powerful tool for the study of the eardrum (and of other parts of the auditory system as well). This paper describes the results obtained so far with a finite-element model of the cat eardrum. The model simulates the behavior of the eardrum in response to uniform pressures of low enough frequencies that inertial and damping effects may be neglected. This corresponds to frequencies below 1 or $2 \mathrm{kHz}$. The model is also restricted to sound pressures low enough that the response of the eardrum is linear.

Section I discusses the finite-element method. Sections II and III then describe the model and the mechanical properties that we have assumed. Section IV presents the results of the model and the effects of parameter variations.

\section{THE FINITE-ELEMENT METHOD}

\section{A. General discussion}

The finite-element method has in the last several years become an extremely widespread engineering tool. Since this is the first time that the method has been applied to the auditory system, we shall give here a very brief outline of the principles involved. The method is fully discussed in a number of textbooks, including Zienkiewicz and Cheung (1967), Zienkiewicz (1971), Desai and Abel (1972), Martin and Carey (1973), Norrie and de Vries (1973), Robinson (1973), Ural (1973), Brebbia and Connor (1974), and Segerlind (1976). In Sec. I B some details of the present implementation which will be meaningful to readers already familiar with the finite-element method are presented.

When using the finite-element method, the physical system to be analyzed is divided into a number of dis- crete two-dimensional or three-dimensional elements which may or may not correspond to natural subdivisions of the actual structure. For example, for an assembly of plates and beams, the elements may consist of the individual plates and beams themselves, if these are easy enough to analyze. On the other hand, a single plate of irregular shape (the eardrum, perhaps) may be considered to be composed of a number of triangular or quadrilateral plate elements which together make up the overall irregular shape, but which separately are easy to analyze. Once one has divided the structure into elements, the mechanical behavior of each element is analyzed, and its response to applied loads is expressed in terms of the displacements of its edges. The result of the element analysis is a matrix equation relating the behavior of the element to the applied forces. The components of the matrix are functions of the shape and properties of the element. One seldom needs actually to do this preliminary analysis, since such analyses have been published in the literature and included in computer programs for a wide variety of element types.

Once the element matrix equations are ready, they are all combined together into one overall system matrix equation. The boundary conditions are also included in the system matrix equation. Since the behavior of each element has been described in terms of its behavior at certain discrete nodes along its edges, this assembly of element matrices is simply a statement of the fact that a node shared by two elements must have the same displacement when considered as part of either element, and of the fundamental assumption that the elements can interact only at these discrete nodes.

The most common alternative to the finite-element method is the finite-difference method, in which one writes down the differential equations governing the whole system, and then replaces the differential operators by difference operators in order to permit a numerical solution. There are a number of features that make the finite-element method particularly attractive. First, in even its simplest form it handles irregular boundary shapes very conveniently. Second, it is rela- 


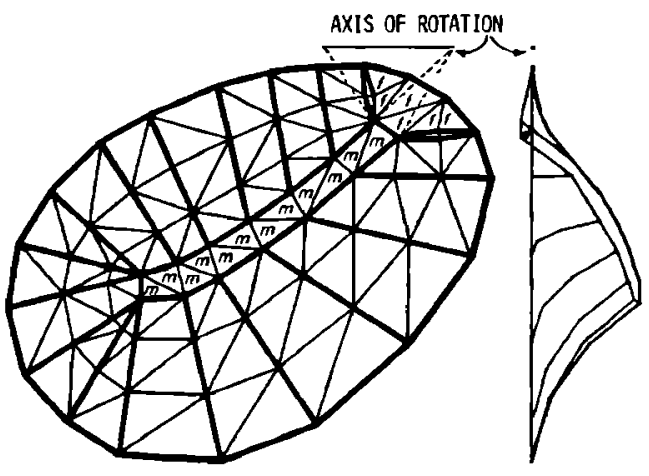

(a)

(b)

FIG. 1. Finite-element model of the cat eardrum. (a) A top view, showing the outlines of the triangular elements. (b) A side view illustrating the curvature of the sides of the drum. The elements corresponding to the pars flaccida are indicated by $f$, the manubrium by $m$. The two elements outlined with dashed lines represent the body of the malleus. The rest of the elements correspond to the pars tensa, except for the four elements separating the pars tensa and pars flaccida, which correspond to part of the tympanic ring.

tively easy to handle inhomogeneities, nonlinearities, and complex geometries and boundary conditions systematically. Third, one need never analyze the behavior of the whole system. All of the analysis is done on simple elements, and even there one need not be able to write down the governing differential equations. The elements can be formulated using any method that is convenient, such as the variational method often used for plates and shells. (A shell is defined as a curved plate.) Another reason for the popularity of the finiteelement method is that it can be understood on a physical, or intuitive, basis. Its use is essentially an extension of the very common technique of analyzing mechanical or electrical systems as networks of interconnected discrete components.

\section{B. Details of this application}

A large number of general-purpose, finite-element computer program packages have been written. Pilkey, Saczalski, and Schaeffer (1974) and Pilkey and Pilkey (1975) are excellent surveys of what is available.

Our model has been implemented using the SAP IV finite-element program, available from the University of California (Bathe, Wilson, and Peterson, 1974). It is written in FORTRAN IV, and has been used on CDC, IBM, and UNIVAC computers. We are using a subset of SAP that we have installed on a DEC PDP-11/70, slightly modified with respect to input and output but computationally unchanged.

We have used the "thin plate and shell" element of SAP (type 6), and also the "boundary" element (type 7). The general thin-shell element is a quadrilateral formed from four flat compatible triangles. We have used the option of specifying our elements directly in terms of the individual triangles. The membrane (in-plane) behavior of the element is represented by the very common constant-strain triangle. The bending behavior is represented by a linear-curvature-compatible triangle with nine degrees of freedom (LCCT-9), which has been described in considerable detail by Clough and Tocher (1966) and Clough and Felippa (1969). A general discussion of the derivation of finite-element approximations to thin-plate mechanics may be found in any textbook on the finite-element method. We have not specified normal rotational stiffnesses since their effect would be very small for the sort of curvatures present in this model (Clough and Wilson, 1971).

The "boundary" element of SAP is a simple one-dimensional spring, either axial or torsional. The element stiffness coefficients are added directly to the total system stiffness matrix.

In the eardrum model discussed here, 120 flat, triangular plate elements are used for the eardrum and manubrium, plus two torsional spring elements representing the stiffness at the ossicular hinge. The model is similar to that of Funnell (1975) and Funnell and Laszlo (1975). The differences are that (1) the position of the axis of rotation has been made more realistic; (2) the ossicular load is now represented by a rotational stiffness about that axis rather than by an artificial stiffness applied to one point on the manubrium; (3) the nodal mesh has been refined by adding more points; and (4) the portion of the annular ligament joining the tympanic ring to the short process of the malleus has been explicitly included, to avoid unreasonably large displacements along the boundary between the pars tensa and pars flaccida.

\section{DESCRIPTION OF MODEL}

\section{A. Geometry}

The essentials of the geometry of the eardrum model are shown in Fig. 1. Figure 1(a) is a view of the drum model in the plane of the annulus, and shows the division into triangular elements. The outlines of the pars tensa, pars flaccida, and manubrium are shown with heavy lines, as are the element boundaries that correspond approximately to the orientations of the radial fibers of the drum. Each of the triangles represents one of the thin-plate elements of SAP. Figure $1(\mathrm{~b})$ is a section through the manubrium, showing the way it points medially.

The position of the ossicular axis of rotation as shown in Fig. $1(\mathrm{a})$ is that given by Khanna (1970, Fig. 21). It is implemented by using two very stiff triangular elements, indicated by dashed lines in the figure; these elements are attached to the upper end of the manubrium, and along the axis of rotation their displacements are all constrained to be zero except for rotation about the axis. The stiffness of the ossicular load is represented by torsional boundary elements. We have assumed for the purposes of this paper that the axis lies in the plane of the tympanic ring. In fact, moving the axis up or down (perpendicular to the plane of the ring) by $0.5 \mathrm{~mm}$ (which represents a quarter of the total 2-mm depth of the eardrum's cone) causes changes of less than $20 \%$ in the calculated vibration amplitudes.

At the time that this work was started, there were no 
good data available to quantify the curvature of the sides of the cone formed by the eardrum. Helmholtz (1869) presented a formula for the shape of the eardrum, but this was based on a theoretical analysis of how the ear drum might function rather than on any quantitative observations of drum shape. Kirikae (1960) suggested a simpler formula, which could be adjusted to fit his relatively crude shape measurements quite well. For even greater simplicity, we have chosen to represent the curvature of the sides of the drum using circular arcs. [Esser (1947) calculated that one of the coefficients in his analysis changed by only $7 \%$ if circular arcs were assumed instead of Helmholtz's curve.] The overall degree of curvature of the eardrum is specified in the models by a dimensionless constant $c$. The radius of curvature of any particular radial fiber $f$ is then given by

$$
r_{f}=c d_{f}
$$

where $d_{f}$ is the straight-line distance between the ends of the fiber. The circular arcs are taken to lie in planes perpendicular to the plane of the tympanic ring.

In the absence of appropriate data, the particular choice of $c$ is more or less arbitrary. We have chosen to set $c$ equal to 1.19 , which is the smallest value that does not result in any nodes of the model lying above the plane of the tympanic ring. The resulting curvature can be seen in Fig. 1(b). In Sec. IV D below we discuss the effects of changing this parameter.

The weakest feature of the geometrical approximation in the model is that each curved radial fiber is approximated by only three straight-line segments. An estimate of the effect of this limited resolution was obtained by using a test case representing a slice out of an infinite curved plate, with a curvature similar to the eardrum model and subjected to a uniform pressure as the eardrum model is. The material thickness and stiffness were the same as for the pars tensa of the eardrum model, and a stiffness was applied to one edge of the shell such that the displacements were similar to those of the drum model. As the number of line segments used to represent the curvature was increased from two to eight, the maximal displacement varied by less than $15 \%$. The displacement of the edge of the shell corresponding to the manubrium, and the volume displacement, varied even less. In view of the uncertainties involved in determining the physical parameters of the model, the subdivision used in obtaining the results presented below is considered to be adequate for the present purposes.

\section{B. Stimulus}

The acoustic stimulation of the eardrum is represented in the finite-element model by a uniform static pressure applied normal to each element. The only exceptions are the two elements joining the manubrium to the axis of rotation, since they correspond to the body of the malleus, behind the eardrum and not directly exposed to the incoming sound. The pressure is speci-

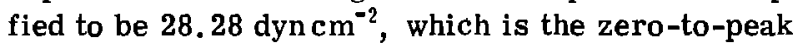
pressure variation equivalent to $100 \mathrm{~dB}$ SPL.

\section{Range of validity}

The range of displacement amplitudes over which this model is valid includes the range of normal hearing. One constraint is that the model is strictly linear, but the middle ear behaves linearly until quite high soundpressure levels. Another constraint common to thinplate finite elements is that the displacements should be much less than the element thickness, in order to permit the simplifying assumption that in-plane and out-of -plane stresses are not coupled. In this case the maximal displacement found at $100 \mathrm{~dB}$ SPL, namely $0.32 \mu \mathrm{m}$, is less than $1 \%$ of the drum thickness $(40 \mu \mathrm{m})$.

The permissible frequency range for the present model is limited by the fact that inertial and damping terms have been completely ignored. This limits the upper frequency to about $1 \mathrm{kHz}$, below which the behavior of the middle ear is essentially independent of frequency (Bêkésy, 1941; Khanna, 1970; and so on). Another potential limitation of the frequency range is due to the assumption of uniformity of the driving soundpressure field. Below $1 \mathrm{kHz}$, however, the wavelength of sound in air is much larger than the dimensions of the eardrum, so uniformity is a reasonable assumption.

\section{MECHANICAL PROPERTIES}

The mechanical properties used in the present models are based on an extensive review of the literature (Funnell, 1975), but it must be admitted that present experimental data are not sufficient to define accurately all of the required parameters. The values used here are rough estimates, and no attempt has been made to optimize the choice of parameter values in order to fit observed vibration amplitudes.

We have assumed the eardrum to be isotropic, homogeneous throughout its thickness, and uniform across its surface. Almost certainly, all of these assumptions are oversimplifications, but they are reasonable first approximations. Not enough data are yet available to completely describe the anisotropies, inhomogeneities, and nonuniformities of the drum in any case.

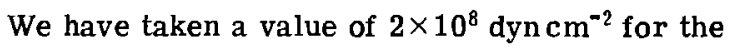
Young's modulus (stiffness) of the pars tensa. This was the value found by Békésy (1949) for a human cadaver eardrum. Other measurements, including those of Kirikae (1960), suggest that this is the right order of magnitude. Note that this is an "effective" stiffness for the combined epidermal, fibrous, and mucosal layers of the drum. The overall thickness of these layers has been taken to be $40 \mu \mathrm{m}$, based on observations by Lim (1968). This thickness is much less than the diameter of the eardrum, so there is no problem with using thinplate finite elements.

The values in the preceding paragraph apply to the pars tensa in particular. For the pars flaccida we have used a stiffness which is much lower, so that it has little effect on the overall behavior of the drum. The manubrium has been assigned a stiffness so high that it is essentially rigid. The part of the annular ring between the pars tensa and pars flaccida has been assigned 
a Young's modulus equal to that of the pars tensa, but has been made $300 \mu \mathrm{m}$ thick.

In the absence of relevant experimental data, the Poisson's ratio for the drum is assumed to be 0.3 . We have shown that the value of this parameter is not very important (Funnell, 1975).

We have assumed that there is no tension in the eardrum in the absence of active middle-ear-muscle contractions. This is contrary to Helmholtz' ideas (1869), but there is no real experimental evident that such tension is essential to eardrum function. It will be seen that tension is not necessary for the present model to explain experimentally observed eardrum displacements.

In the model considered here, we have assumed the eardrum to be "fully clamped" at its attachments to the annular ligament and to the manubrium. That is, all displacements and rotations are constrained to be zero (with respect to the supporting structure) at these boundaries, as opposed to a "simply supported" condition, where only the displacements are constrained to be zero. There is no strong experimental evidence to support this assumption. However, we have shown that it makes little difference which type of support is adopted (Funnell, 1975).

The loading of the ossicular chain and cochlea on the eardrum is represented in our model as a single effective torsional stiffness acting at the "hinge" of the manubrium. There are no data available which provide an estimate for the stiffness of the ossicular chain separate from that of the eardrum. The middle-ear circuit models presented by Zwislocki (1963), Peake and Guinan (1967), Funnell (1972), and others do use parameter values for this quantity, but they are based strictly on empirical curve fitting with models whose eardrum representations are very crude. Consequently, as a first approximation, we have made an orderof-magnitude estimate of the ossicular load based on various data. The most important contribution appears to be from the input impedance of the cochlea. Lynch, Nedzelnitsky, and Peake (1976) found that the total cochlear input impedance is capacitive at low frequencies, with a value of about $5.6 \times 10^{6}$ acoustic ohms at $100 \mathrm{~Hz}$. Assuming a stapedial-footplate area of 0.012 $\mathrm{cm}^{2}$ (Wever and Lawrence, 1954), this corresponds to a mechanical impedance of about $51 \times 10^{4} \mathrm{dyn}^{-1}$. Taking the incudal lever arm to be $0.19 \mathrm{~cm}$ (again from Wever and Lawrence) gives an effective angular stiffness of about $18 \times 10^{3}$ dyn $\mathrm{cm}$ due to the cochlea.

To estimate the stiffness due to the suspensory structures of the incus and malleus, we have used oversimplified geometrical models. For the posterior incudal ligament, we have calculated the stiffness of a cylindrical elastic bushing with inner and outer diameters of 0.05 and $0.15 \mathrm{~cm}$, a length of $0.05 \mathrm{~cm}$, and material properties the same as the pars tensa; dividing the resultant stiffness by four to account for the fact that the ligament does not completely surround the posterior process of the incus gives an angular stiffness of 8500 dyn $\mathrm{cm}$. Modeling the anterior process of the malleus as a uniform rod, fully clamped to the temporal bone at one end and to the body of the malleus at the other, with diameter $0.01 \mathrm{~cm}$ and length $0.3 \mathrm{~cm}$, and a stiffness of $10^{11} \mathrm{dyn} \mathrm{cm}^{-2}$, gives an angular stiffness of only 126 dyn $\mathrm{cm}$. The nonfibrous tissue surrounding this process appears to contribute even less stiffness.

The total calculated angular stiffness due to the cochlea and posterior ligament is thus $26.8 \times 10^{3}$ dyn $\mathrm{cm}$. To account for small additional stiffnesses of other attached structures, we have used an overall value of $28 \times 10^{3}$ dyn $\mathrm{cm}$.

We have neglected the loading effect of the middle-ear air cavities in this model. Inclusion of the cavities can be expected to reduce the manubrial displacements by about $5 \mathrm{~dB}$ (Peake and Guinan, 1967). Preliminary study of the plane-membrane model presented in Funnell (1975) suggests that the form of the displacement pattern (apart from its absolute amplitude) is not greatly affected by the cavities at low frequencies.

\section{RESULTS}

\section{A. Resufts for initial parameter values}

Figure 2 shows displacement contours calculated for the cat model using the parameter values specified above. The vibration pattern is very similar to that observed experimentally by Khanna (1970). The drum displacements are greater than those of the manubrium: There is an amplitude maximum in the posterior region, and a smaller local maximum in the anterior region.

Apart from the general shape of the contour lines, two quantitative measures to use in comparing the experimental and the calculated results are (1) the amplitude of the maximal drum displacement, and (2) the ratio of that displacement to the displacement of the tip of the manubrium. The former is a measure of the

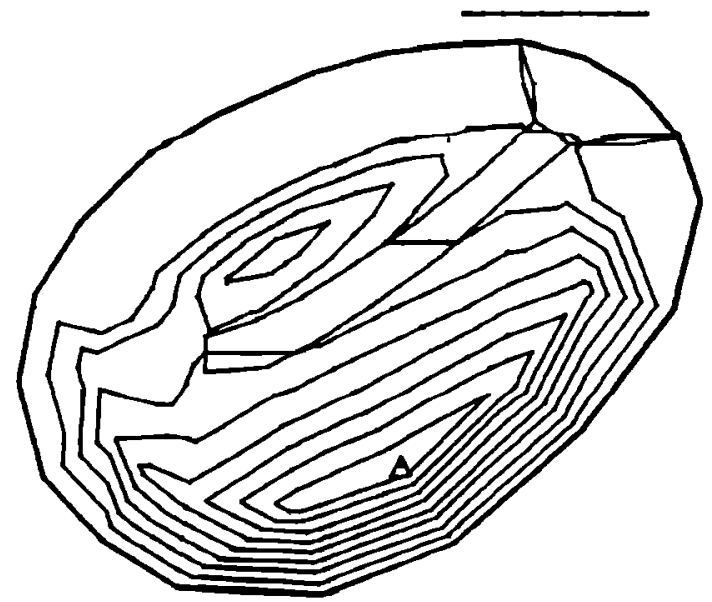

FIG. 2. Vibration pattern calculated with the model. The contour lines are lines of constant vibration amplitude, equally spaced on an amplitude scale. Only displacements in the $z$ direction are considered, which corresponds to the component of displacement measured in the holographic experiments of Khanna and Tonndorf. The small triangle represents the point of maximal vibration amplitude, corresponding to $0.32 \mu \mathrm{m}$ for a low-frequency pure-tone input of $100 \mathrm{~dB}$ SPL. 


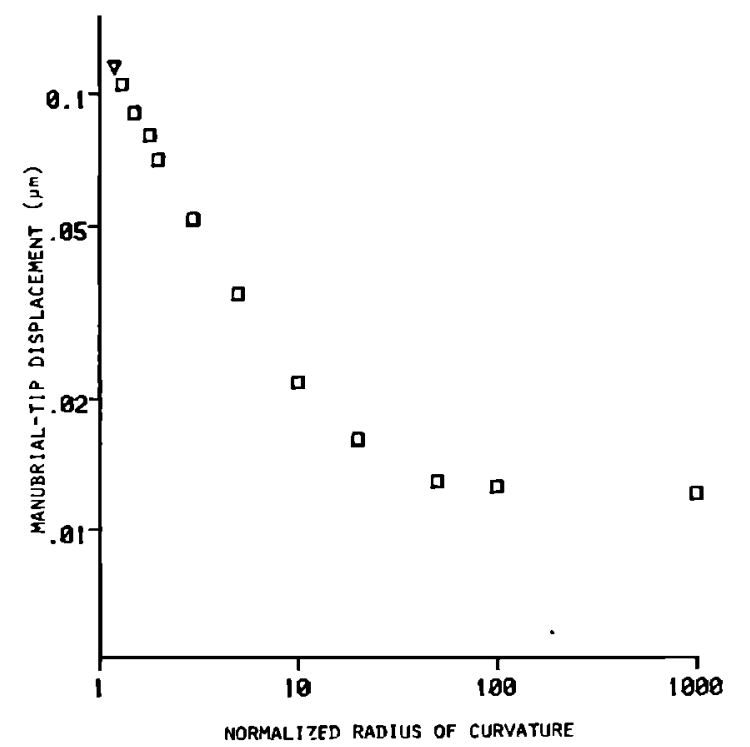

FIG. 3. Effect of curvature. The displacement of the tip of the manubrium is plotted against the degree of curvature of the sides of the drum. The inverted triangle represents the behavior at the normal curvature. As the radius of curvature is increased until the sides of the drum are essentially straight, the manubrial displacement decreases steadily.

overall displacement of the drum, and the latter is a dimensionless measure of the coupling of the drum to the ossicles and of the degree of sound transmission to the middle ear. Tonndorf and Khanna (1971) reported a maximal drum displacement of $1.5 \mu \mathrm{m}$ at $600 \mathrm{~Hz}$ and $111 \mathrm{dBSPL}$. This is equivalent to $0.42 \mu \mathrm{m}$ at $100 \mathrm{~dB}$ SPL. (The frequency of $600 \mathrm{~Hz}$ is low enough that the displacement is practically the same as at $0 \mathrm{~Hz}$.) By comparison, the results presented in Fig. 2 show a maximal drum displacement of $0.32 \mu \mathrm{m}$ at $100 \mathrm{~dB}$ SPL.

Khanna and Tonndorf (1972) report a ratio of peak displacement/tip displacement equal to 3 at low frequencies. The computed results in Fig. 2 have a ratio equal to 2.8 .

\section{B. Variation of drum material properties}

Doubling the Young's modulus of the pars tensa reduced the maximal drum displacement by about $48 \%$; cutting the stiffness in half increased the maximal displacement by $79 \%$. Similarly, doubling and halving the thickness of the pars tensa changed the maximal drum displacement by $-64 \%$ and $120 \%$, respectively. In all four cases, however, the vibration pattern remained qualitatively the same, which is reflected in the fact that the largest concomitant change in the peak/tip displacement ratio was only about $17 \%$.

\section{Variation of ossicular-hinge stiffness}

Doubling the stiffness of the ossicular hinge decreased the maximal displacement by only $11 \%$; halving it increased the displacement by $6 \%$. The form of the vibration pattern was little changed, and the ratio peak/tip changed by $8 \%$ and $-4 \%$, respectively.

\section{Variation of curvature}

Increasing the curvature parameter $c$, that is, straight. ening the radial fibers of the drum, increases the maximal drum displacement by up to $48 \%$. The peak/ tip displacement ratio continues to increase indefinitely. A more meaningful parameter to look at in this case is the actual displacement of the manubrium, which is a direct measur 2 of how well the eardrum couples the applied sound opressure to the middle ear. Figure 3 shows that the displacement of the tip of the manubrium decreases markedly as the fibers straighten.

The increase of peak displacement with increasing radius of curvature, and the consequent inability to couple sound-pressure changes to the malleus, are expected on the hasis of normal shell behavior: A nearly flat shell cannot use its in-plane stiffness to resist transverse loads, and its bending stiffness will be low if the shell is thin. Thus the effective stiffness of the drum itself becomes small compared to that of the ossicular load, and the pressure variations are most easily accommodated by deforming the drum, without displacing the manubrium very much.

\section{E. Variation of depth of cone}

The previous section showed that the nonzero curvature of the sides of the cone formed by the eardrum could aid in transmitting sound to the ossicles. One might also wonder if the conical shape itself has some functional significance. Figure 4 shows the effects on manubrial displacement of changing the depth of the cone. This wis done by scaling the $z$ coordinates of each point in the model by a "relative depth" factor.

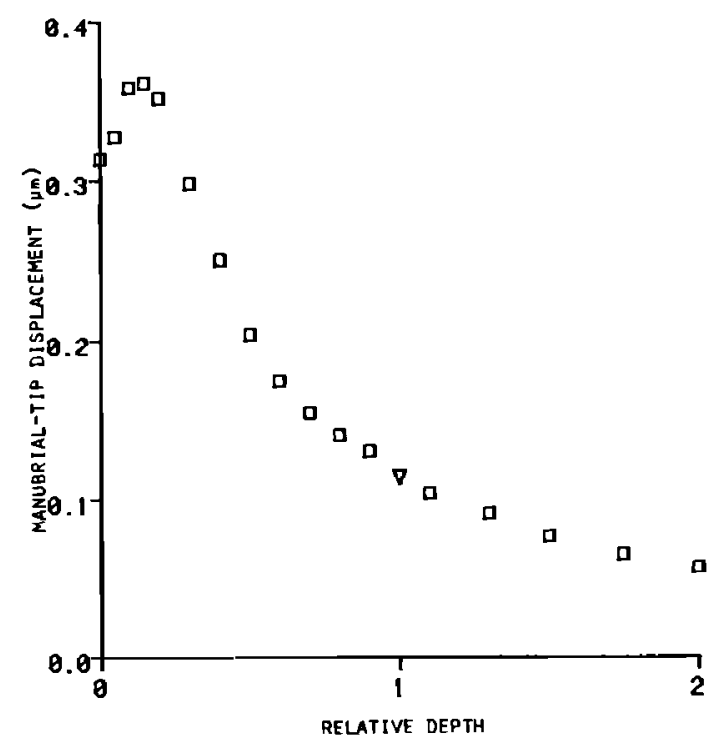

FIG. 4. Effect of depth. The displacement of the tip of the manubrium is plotted agalnst the depth of the cone formed by the drum (normelized with respect to its normal depth). As in Fig. 3, the inverted triangle represents the normal behavior. Except when the drum is very close to being completely flat, the manubrial displacement decreases as the depth is increased. The discrepaney for very flat shapes may be a numerical problem associated with the resulting very small curvatures. 
Note that the circular arcs formed by the radial fibers become elliptical under this operation. Increasing the depth of the cone reduces the displacement of the manubrium, that is, reduces the ability of a given soundpressure level to move the ossicles. This presumably is because the deeper conical shapes impart greater stiffness to the eardrum, just as argued (albeit in favor of the conical shape) by Békésy (1941) in connection with his hinged-plate model.

It would thus appear that the conical shape is disadvantageous, assuming that the eardrum actually functions as modeled here. One might speculate, however, that the conical shape provides an "adequate" middleear sensitivity while permitting low eardrum mass, and thus good high-frequency response. If the drum were flat, or a very shallow cone, then a given degree of sensitivity would require a stiffer (and thus heavier) drum than if it were a deeper cone. Gran (1968) found that a stiffness of 60-250 times the true value was required to get reasonable displacements with a planeplate model.

It is also possible that the conical shape is important in conjunction with the shape of the terminal portion of the ear canal and the inclination of the drum with respect to the canal.

\section{F. Effect of anisotropy}

Since the model is quite successful using isotropic material properties, it has not been necessary to introduce anisotropy, and it does not seem worthwhile at the moment to do a detailed study of the effects of anisotropy in view of the number of questions still unresolved concerning eardrum properties and behavior. However, this section discusses a single example of displacements calculated with an anisotropic model, to give an idea of what may be expected.

In line with assumptions of Helmholtz (1869) and Esser (1947), the radial fibers have been assumed to be much less extensible than the circular fibers. In particular, the Young's modulus in the radial direction (as defined by the element boundaries shown in Fig. 1) has been left at the value of $2 \times 10^{8} \mathrm{dyn} \mathrm{cm}^{-2}$ used before, while the Young's modulus in the perpendicular direction (in each element) has been reduced to $10^{6} \mathrm{dyn} \mathrm{cm}^{-2}$. The two Poisson's ratios, and the shear modulus, have been set of zero, equivalent to assuming that the radial $\mathrm{fi}-$ bers slide over one another without lateral interaction. This assumption is open to question, but there are no experimental data available to decide the issue. The vibration pattern under these conditions has basically the same shape as with isotropic material properties, but the displacements are 2.5 to 3 times larger. This indicates that the drum would be more effective in transmitting vibrations to the ossicles if it were anisotropic in the way described here. This expectation was, of course, what prompted Helmholtz's original hypothesis that the circular fibers were much more extensible than the radial fibers: The latter are in an ideal position to transmit forces to the manubrium, while the former can only interfere with this action.

\section{CONCLUSIONS}

Using physiologically reasonable parameter values, the shell model of the cat eardrum yields results quite close to previous experimental findings. The same is true of guinea pig and human models discussed in Funnell (1975), although the experimental data are less satisfactory in those cases. On this basis, one can conclude that presently available experimental evidence is consistent with the hypothesis that the eardrum is basically equivalent to an isotropic thin (curved) shell. It is not necessary at this time to postulate either resting tension in the drum or anisotropy (that is, differences in properties between the radial and circular fibers).

The results described here suggest that the most important determinants of low-frequency eardrum behavior include material stiffness and thickness, curvature and conical shape, and anisotropy. Boundary conditions, Poisson's ratio, ossicular loading, and air loading are less important. These conclusions affect the direction of further efforts to understand the mechanical functioning of this system. Quantitative data on the shape of the eardrum have recently become available (Khanna and Tonndorf, 1975) which will help to refine the model considerably.

\section{ACKNOWLEDGMENTS}

The thesis work on which this paper is partly based was supported by the Medical Research Council of Canada, le Ministère de l'Education du Quếbec, and the McConnell Foundation. Subsequent work has been supported by the Macdonald-Stewart Foundation and the Medical Research Council of Canada.

Bathe, K. -J., Wilson, E. L., and Peterson, F. E. (1974). "SAP IV. A Structural Analysis Program for static and dynamic response of linear systems," Report No. EERC 73-11 (University of California, Berkeley), vii +59 pp. + appendices.

Békésy, G. v. (1941). "On the measurement of the amplitude of vibration of the ossicles with a capacitive probe," Akust. Z. 6, 1-16 (in German).

Békésy, G. v. (1949). "The structure of the middle ear and the hearing of one's own voice by bone conduction," J.

Acoust. Soc. Am, 21, 217-232.

Brebbia, C. A., and Connor, J. J. (1974). Fundamentals of Finite Element Techniques for Structural Engineers (Wiley, New York), 269 pp.

Clough, R. W., and Tocher, J. L. (1966). "Finite element stiffness matrices for analysis of plate bending," in Proc. Conf. Matrix Methods in Structural Mechanics, edited by J. S. Przemieniecki, R. M. Bader, W. F. Bozich, J. R. Johnson, and W. J. Mykytow (Wright Patterson A FB, OH), Report No. A F FDL-TR-66-80, pp. 515-545.

Clough, R. W., and Felippa, C. A. (1969). "A refined quadrilateral element for analysis of plate bending," in Proc. 2 nd Conf. Matrix Methods in Structural Mechanics, edited by $L$. Berke, R. M. Bader, W. J. Mykytow, J. S. Przemieniecki, and M. H. Shirk (Wright Patterson AFB, OH), Report No. AFFDL-TR-68-150, pp. 399-440.

Clough, R. W., and Wilson, E. L. (1971). "Dynamic finite element analysis of arbitrary thin shells, "Comput. Struct. 1(1), 33-56. 
Desai, C. S., and Abel, J.F. (1972). Introduction to the Finite Element Method. A numerical method for engineering analysis (Van Nostrand Reinhold, New York).

Esser, M. H. M. (1947). "The mechanism of the middle ear: II. The drum," Bull. Math. Biophys. 9(2), 75-91.

Funnell, W. R. J. (1972). "The Acoustical Impedance of the Guinea-Pif Middle Ear and the Effects of the Middle-Ear Muscles," M. Eng. thesis (McGill University, Montreal), vi +93 pp. Available from the author.

Funnell, W. R. J. (1975). "A Theoretical Study of Eardrum Vibrations Using the Finite-Element Method," Ph.D. thesis (McGill University, Montreal), $x+199$ pp., updated 1976. Available from the author, or on microfilm from the National Library of Canada.

Funnell, W. R. J., and Laszlo, C. A. (1974). "Simulating the behavior of the eardrum by the finite-element method," $\mathrm{J}$. Acoust. Soc. Am. 56, S 3(A).

Funnell, W. R. J., and Laszlo, C. A. (1975). "Modeling the eardrum as a doubly curved shell using the finite-element method," J. Acoust. Soc. Am. 67, S72(A).

Gran, S. (1968). "The analytical basis of middle-ear mechanics. A contribution to the application of the acoustical impedance of the ear," Dissertation (Univ. Oslo), iv $+63 \mathrm{pp}$. (in German).

Helmholtz, H. L. F. (1869). "The mechanism of the middleear ossicles and of the eardrum," Pflügers Arch. Physiol. (Bonn) 1, 1-60 [in German: transl. by A. H. Buck and N. Smith (W. Wood, New York, 187369 pp.) and by J. Hinton, (Publ. New Sydenham Soc. London 62, 97-155 (1874)].

Khanna, S. M. (1970). "A. Holographic Study of Tympanic Membrane Vibrations in Cats," Ph. D. thesis (City University of New York, New York), 214 pp. (unpublished).

Khanna, S. M., and Tonndorf, J. (1972). "Tympanic membrane vibrations in cats studied by time-averaged holography," J. Acoust. Soc. Am. 51, 1904-1920.

Khanna, S. M., and Tonndorf, J. (1875). "Tympanic mem-" brane shape determined by moiré topography," J. Acoust. Soc. Am. 57, S72(A).

Kirikae, I. (1960). The Structure and Function of the Middle Ear (Univ. Tokyo Tokyo), vi $+157 \mathrm{pp}$.
Lim, D. J. (1968). "Tympanic membrane. Electron microscopic observation. Part I. Pars tensa," Acta Oto-Laryngol. 66, 181-198.

Lynch, T. J., III, Nedzelnitsky, V., and Peake, W. T. (1976). "Measiurements of acoustic input impedance of the cochlea in catsi," J. Acoust. Soc. Am. 59, S30(A).

Martin, H. C., and Carey, G. F. (1973). Introduction to Finite Element Analysis. Theory and application (McGraw-Hill, New York), xi..i +386 pp.

Norrie, D. H. , and de Vries, G. (1973). The Finite Element Method. Fundamentals and applications (Academic, New York), xiii $+322 \mathrm{pp}$.

Peake, W. T., and Guinan, J. J., Jr, (1967). "A circuit model for the cat's midddle ear." MFT Lab. Electron. Q. Prog. Rep. $84,320-326$.

Pilkey, W. D., Saczalski, K., and Schaeffer, H. G. (Eds.) (1974). Structural Mechanics Computer Programs-Surveys. Assessments, and Availability (U. P. Virginia, Charlottesville, VA), xiii-110 $5 \mathrm{pp}$.

Pilkey, W. D. c.nd Pilkey, B. F. (Eds.) (1975). Shock and Vibration Computer Programs. Reviews and Summaries (Shock and Vibration Information Center Nav. Res. Lab., Washington, D. C.), viii +663 pp.

Robinson, J. (1973). Integrated Theory of Finite Element Methods (Wiley, London), xxi $+428 \mathrm{pp}$.

Segerlind, L. J. (1976). Applied Finite Element Analysis (Wiley, New York), xiii +422 pp.

Tonndorf, J., and Khanna. S. M. (1971). "The tympanic membrane as a part of the middle ear transformer, "Acta OtoLaryngol. 71, 177-180.

Ural, O. (1973). Finite Element Method. Basic concepts and applications (Intext Educational, New York), xiii +272 pp.

Wever, E. G. and Lawrence, M. (1954). Physiological Acoustics (Princetor! U. Princeton, NJ), xii $+454 \mathrm{pp}$.

Zienkiewicz, O. C., and Cheung, Y. K. (1967). The Finite Element Method in Structural and Continuum Mechanics (McGraw-Hill: London).

Zienkiewicz, O. C. (1971). The Finite Element Method in Engineering Science (McGraw-Hill, London), xiv + 521 pp. Zwislocki, J. (1.963). "Analysis of the middle ear function. Ш. Guinea-pig ear," J. Acoust. Soc. Am 35, 1034- 1040. 